\title{
Inhalt des Jahrgangs 39 / Sommaire du volume 39
}

J.Z.Baruch, Maimonides as a Physician

Luigi Belloni, Il Morgagni tra il Malpighi e il Cotugno

Peter Bischoff und Peter Speiser, Mittelalterliche Augenheilkunde im Kloster St. Gallen

Jakob Büchi, Die wiederaufgefundene «Pharmacia Contracta» des

Johann Jakob Scheuchzer (1672-1733)

Robert W. Carrubba, The Latin Document Confirming the Date and Institution of Wilhem ten Rhyne's M.D.

Willem F.Daems, Renward Cysat und Paracelsus

Adolf Faller, Die Präparation der weißen Substanz des Gehirns bei Stensen, Willis und Vieussens

- Die «Tetralogie von Fallot». Zur geschichtlichen Entwicklung eines kongenitalen Herzsyndroms von Niels Stensen bis zur modernen Herzchirurgie

Adolf M. Fehr, Geschichte des Winterthurer Spitals

Klaus-Dietrich Fischer, Antike Verse in medizinischen Schriften des Mittelalters

Beat Glaus, Autographen und Manuskripte zur ETH- und zur Wissenschaftsgeschichte

Hermann Hörger, Krankheit und religiöses Tabu - die Lepra in der mittelalterlich-frühneuzeitlichen Gesellschaft Europas

Alfred Huber, Walter Rudolf Hess als Ophthalmologe

Huldrych M. Koelbing, Editorial: Zur Entwicklung der Schweizer Spitäler und Heilanstalten im Vergleich mit Nachbarländern

François Ledermann, La thérapeutique médicamenteuse et la psychiatrie allemande du XIX ${ }^{\mathrm{e}}$ siècle: concordances, oppositions, indifférence?

Karl Lenggenhager, Zur Schlichtung des Streites zwischen Haldane und Mosso bezüglich der Höhen-Hypokapnie

Zdenko Levental, Medizinisches in den Schriften Edward Brownes (1642-1708)

Mary Lindemann, Fürsorge für arme Wöchnerinnen in Hamburg um 1800: die Beschreibung eines «Entbindungs-Winkels»

Stefan Loretan, Das Antoniusspital in Brig

Karl von Meyenn, Die Rezeption der Wellenmechanik und Schrödingers Reise nach Amerika im Winter 1926/27

- Theoretische Physik in den dreißiger Jahren. Entwicklung einer Wissenschaft unter ideologischen Zwangsbedingungen

Axel Hinrich Murken, Grundzüge des deutschen Krankenhauswesens von 1780 bis 1930 unter Berücksichtigung von Schweizer Vorbildern

Walter Nussbaum, Die Grippe-Epidemie 1918/1919 in der schweizerischen Armee

Friedrich Rintelen, Zur Persönlichkeit Karl Gustav Jungs

- Von der Augenheilanstalt zur Universitätsaugenklinik in Basel

Hans Peter Schönwetter, Zur Geschichte der Akromegalie

Eduard Seidler, Die Behinderten in Herten

Peter Sommer, Die ersten Jahrzehnte des Jenner-Kinderspitals in Bern

Werner H.Stöcklin, Sakpata, ein Beitrag afrikanischer Medizinmänner zur Pockeneradikation

Delfo Tesi, Augustin-Pyramus de Candolle: Essai d'élaboration d'une taxonomie théorique au XIX ${ }^{\mathrm{e}}$ siècle

Ulrich Tröhler, Britische Spitäler und Polikliniken als Heil- und Forschungsstätten $1720-1820$ 
Vera Waldis, Hospitalisation und Absonderung in Pestzeiten - die Schweiz im Vergleich zu Oberitalien

Hans H. Walser, Der Weg der Schweizer Psychiatrie ins zwanzigste Jahrhundert $\quad 97$

Peter G. Walser, Walter Rudolf Hess

Buchbesprechungen

József Antall, Bilder aus der Geschichte der europäischen Heilkunde und Pharmazie (Urs Boschung)

Gesine Asmus (Hg.), Hinterhof, Keller und Mansarde (Erwin H. Ackerknecht)

Juan José Barcia Goayanes, Onomatologia Anatomica Nova Bd. IV

(Erwin H. Ackerknecht)

Leonard Barkan, Nature's Work of Art: The Human Body as Image of the World (Balz Engler)

Arnold Bauer, Rudolf Virchow, der politische Arzt (Erwin H. Ackerknecht)

Jakob Büchi, Die Entwicklung der Rezept- und Arzneibuchliteratur, 1.Teil: Altertum und Mittelalter (H. R. Fehlmann)

W.F.Bynum, V.Nutton, Theories of Fever from Antiquity to the Enlightenment

(Erwin H. Ackerknecht)

Johannes Dryander, Vom Eymser Bade... (Erwin H. Ackerknecht)

D. von Engelhardt und H.Schipperges, Die inneren Verbindungen zwischen Philosophie und Medizin im 20. Jahrhundert (Carl Haffter)

Hans Err (Hg.) Schmökern rezeptfrei (Marie-Louise Portmann)

Ludwig Flek, Genesis and Development of a Scientific Fact (Erwin H. Ackerknecht)

Karl Garbers, Ishaq Ibn Imran, Abhandlung über die Melancholie, und

Constantini Africani Libri Duo De Melancholia (Carl Haffter)

Karl S. Guthke, Haller im Halblicht (Urs Boschung)

Arthur E. Imhof, Die gewonnenen Jahre (Erwin H. Ackerknecht)

Danielle Jacquart, Le Milieu Médical en France du XII ${ }^{\mathrm{e}}$ au XV ${ }^{\mathrm{e}}$ Siècle

(Erwin H.Ackerknecht)

Ilse Jahn, Charles Darwin (Marie-Louise Portmann)

Brian Lawn, The Prose Salernitan Questions, edited from a Bodleian Manuscript (Carl Haffter)

Erna Lesky, Meilensteine der Wiener Medizin. Große Ärzte Österreichs in drei Jahrhunderten (Huldrych M. Koelbing)

Renato Giuseppe Mazzolini, The Iris in Eighteenth-century Physiology (Urs Boschung)

Renato G. Mazzolini und Giuseppe Ongaro (Hg.), Carteggio con Leopoldo Marc'Antonio Caldani, 1758-1794 (Urs Boschung)

V.C.Medvei, A History of Endocrinology (Erwin H. Ackerknecht)

R. Klaus Müller, Bo Helmstedt, Karlheinz Lohs (Hg.), Der Toxikologe Louis Lewin (Erwin H. Ackerknecht)

Axel Hinrich Murken, Die bauliche Entwicklung des deutschen Allgemeinen Krankenhauses im 19. Jahrhundert (Erwin H. Ackerknecht)

Pharmaziehistorischer Kongreß Basel, Vorträge (Willem F. Daems)

Philippe Pinel, The Clinical Training of Doctors: an Essay of 1793 (Esther Fischer-Homberger)

Shirley A.Roe, Matter, Life, and Generation. Eighteenth-century embryology and the Haller-Wolff debate (Urs Boschung)

Karl Ed.Rothschuh, Iatromagie. Begriff, Merkmale, Motive, Systematik (Carl Haffter)

Hanspeter Ruesch, Lebensverhältnisse in einem frühen schweizerischen Industriegebiet (Guido Sigron) 
Christoph Sachße, Florian Tennstedt, Geschichte der Armenfürsorge in Deutschland. Vom Spätmittelalter bis zum Ersten Weltkrieg (Erwin H. Ackerknecht)

Heinrich Schipperges, Weltbild und Wissenschaft. Eröffnungsreden zu den Naturforscherversammlungen 1822 bis 1972 (Carl Haffter)

Heinrich Schipperges, Arabische Medizin im lateinischen Mittelalter (Carl Haffter)

Wilfried Schröder, Disziplingeschichte als wissenschaftliche Selbstreflektion der historischen Wissenschaftsforschung (Heinz Balmer)

Martin Schwarzbach, Auf den Spuren unserer Naturforscher (Heinz Balmer)

Short Title Catalogue of Eighteenth Century Printed Books in the National Library of Medicine (Urs Boschung)

Florian Tennstedt, Sozialgeschichte der Sozialpolitik in Deutschland. Vom 18. Jahrhundert bis zum Ersten Weltkrieg (Erwin H. Ackerknecht)

Claude Alexandre Thomasset, Placides et Timéo ou Li secrés as philosophes / Une vision du monde à la fin du XIII ${ }^{\mathrm{e}}$ siècle. Commentaire du dialogue de Placides et Timéo (Carl Haffter)

L. Pecorella Vergnano, L.Belloni, M.L.Turchetti Grossi, Libri e Manoscritti di Haller (Urs Boschung)

\section{Inhalt / Sommaire}

Adolf Faller, Die «Tetralogie von Fallot». Zur geschichtlichen Entwicklung eines kongenitalen Herzsyndroms von Niels Stensen bis zur modernen Herzchirurgie

J.Z.Baruch, Maimonides as a Physician

Karl Lenggenhager, Zur Schlichtung des Streites zwischen Haldane und Mosso bezüglich der Höhen-Hypokapnie

Hans Peter Schönwetter, Zur Geschichte der Akromegalie

Mary Lindemann, Fürsorge für arme Wöchnerinnen in Hamburg um 1800: die Beschreibung eines «Entbindungs-Winkels»

Werner H.Stöcklin, Sakpata, ein Beitrag afrikanischer Medizinmänner zur Pockeneradikation

Karl von Meyenn, Theoretische Physik in den dreißiger Jahren.

Die Entwicklung einer Wissenschaft unter ideologischen Zwangsbedingungen

Beat Glaus, Autographen und Manuskripte zur ETH- und zur Wissenschaftsgeschichte

Klaus-Dietrich Fischer, Antike Verse in medizinischen Schriften des Mittelalters

François Ledermann, La thérapeutique médicamenteuse et la psychiatrie allemande du XIX ${ }^{\mathrm{e}}$ siècle: concordances, oppositions, indifférence?

Willem F.Daems, Renward Cysat und Paracelsus

Robert W. Carrubba, The Latin Document Confirming the Date and Institution of Wilhem ten Rhyne's M.D. 


\section{Buchbesprechungen}

V.C.Medvei, A History of Endocrinology (Erwin H. Ackerknecht)

Arthur E. Imhof, Die gewonnenen Jahre. Von der Zunahme unserer Lebensspanne seit 300 Jahren oder von der Notwendigkeit einer neuen Einstellung

zu Leben und Sterben (Erwin H. Ackerknecht)

Juan José Barcia Goayanes, Onomatologia Anatomica Nova,

Historia del Lenguaje Anatomico Bd. IV (Erwin H. Ackerknecht)

Arnold Bauer, Rudolf Virchow, der politische Arzt (Erwin H. Ackerknecht)

Gesine Asmus (Hg.), Hinterhof, Keller und Mansarde. Einblicke in

Berliner Wohnungselend 1901-1920 (Erwin H. Ackerknecht)

R. Klaus Müller, Bo Helmstedt, Karlheinz Lohs (Hg.), Der Toxikologe Louis Lewin (Erwin H. Ackerknecht)

Johannes Dryander, Vom Eymser Bade... (Erwin H. Ackerknecht)

W.F.Bynum, V. Nutton, Theories of Fever from Antiquity to the Enlightenment

(Erwin H. Ackerknecht)

Danielle Jacquart, Le Milieu Médical en France du XII ${ }^{\mathrm{e}}$ au XV $\mathrm{XV}^{\mathrm{e}}$ Siècle (Erwin H. Ackerknecht)

Leonard Barkan, Nature's Work of Art: The Human Body as Image of the World (Balz Engler)

Erna Lesky, Meilensteine der Wiener Medizin. Große Ärzte Österreichs in drei Jahrhunderten (Huldrych M. Koelbing)

Die Vorträge des Internationalen Pharmaziehistorischen Kongresses Basel 1979 (Willem F.Daems)

Philippe Pinel, The Clinical Training of Doctors: an Essay of 1793. Edited and translated, with an introductory essay by Dora B. Weiner.

Baltimore, The Johns Hopkins University Press, 1980 (Esther Fischer-Homberger)

Jakob Büchi, Die Entwicklung der Rezept- und Arzneibuchliteratur, 1.Teil: Altertum und Mittelalter (Hans Rudolf Fehlmann)

Heinrich Schipperges, Arabische Medizin im lateinischen Mittelalter (Carl Haffter)

Karl Garbers, Ishaq Ibn Imran, Abhandlung über die Melancholie, und Constantini Africani Libri Duo De Melancholia (Carl Haffter)

Brian Lawn, The Prose Salernitan Questions (Carl Haffter)

Claude Alexandre Thomasset, Placides et Timéo ou Li secrés as philosophes / Une vision du monde à la fin du XIII ${ }^{\mathrm{e}}$ siècle.

Commentaire du dialogue de Placides et Timéo (Carl Haffter)

Karl Ed.Rothschuh, Iatromagie. Begriff, Merkmale, Motive, Systematik (Carl Haffter)

Beiträge zur schwäbischen Literatur- und Geistesgeschichte (Carl Haffter)

D. von Engelhardt und H. Schipperges, Die inneren Verbindungen zwischen Philosophie und Medizin im 20. Jahrhundert (Carl Haffter)

Karl S. Guthke, Haller im Halblicht (Urs Boschung)

A Short Title Catalogue of Eighteenth Century Printed Books in the National Library of Medicine (Urs Boschung)

Shirley A.Roe, Matter, Life, and Generation. Eighteenth-century embryology and the Haller-Wolff debate (Urs Boschung)

Wilfried Schröder, Disziplingeschichte als wissenschaftliche Selbstreflexion der historischen Wissenschaftsforschung (Heinz Balmer) 